Historic, Archive Document

Do not assume content reflects current scientific knowledge, policies, or practices. 



\section{Kirchhoff's Wholesale Price List "Gladioli of Unusual Merit" SPRING 1930}

TERMS: 25 Bulbs at 100 Rate, 250 Bulbs at 1000 Rate, Minimun Order \$5.00, Prices Net, F.O.B. Wilson, N. Y. Orders reserved for shipment as late as April 15th, when accompanied by a deposit of at least 25 per cent.

\begin{tabular}{|c|c|c|}
\hline No. 1 & No. 2 & No. 3 \\
\hline $11 / 2$ in. up & $1^{1}+-1^{1} \geq$ & $1-1_{t}^{1}$ \\
\hline $100 \quad 1000$ & $100 \quad 1000$ & 100 \\
\hline
\end{tabular}

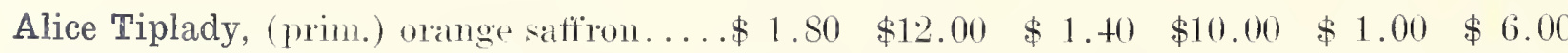

America, soft pink.

Anna Eberius, dalli relvety purple..... 2.50

Appleblossom, (Kairehhofl"s)

B. L. Smith, lavender.

16.00

12.00

20.00

$1+0.00$

4.00

3.5 .00

Bengal Tiger, mottled.

3.00

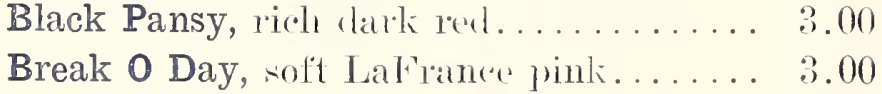

3.00

25.00

Carmen Sylva, white.

25.00

Chalice Flower, white suffused with

$+.00$

2.50

Chateau Thierry, bright scallet red

Crimson Glow, bright crinson.

2.00

5.00

Diana tall crimson

2.50

Diener's White, extrat rigorous white.... 8.00

Dorothy Wheeler, (prim)

Dr. Bennett, vivid flame red.

2.00

6.00

16.00

50.00

Dr. Van Fleet, low pink

2.50

3.50

30.00

Early Sunrise, coral rerl, early.

3.00

25.00

E. J. Shaylor, cerise, ruffled

2.00

E. Kirtland, tall pink.

3.00

E. Miller, (Kirchliofe"s) coral pink

5.00

Fern Kyle, ruffled white.

$+.00$

Flaming Sword, brilliant medium red.... 3.00

Fontaine, rulted light pink......... 4.00

Geraldine Farrar, light hlue......... 10.00

Giant Nymph, shrimp pink.......... …

Gold, clear vellow.............. 3.00

Golden Dream, golden yellow. . . . . . . . 20.00

Golden Measure, rellow large. . . . . . . . . 4.00

Halley, early salnon.............. 1.80

Herada, mauve................ 2.00

J. A. Carbone, iridercent orange. . . . . . . 2.50

14.00

20.00

16.00

$+5.00$

....

.....

....

....

$\ldots$

$\ldots .$.

....

$\ldots \ldots$

3.5 .00

14.00

15.00

20.00
1. 40

1.80

$1+.00$

$: 300$

2.50

2.50

2.50

2.50

3.00

2.00

1.50

$+.00$

2.00

1.50

万. 00

2.00

2.5

1.50

2.00

$+.00$

3.00

ㄴ.

3.00

8.00

2.00

2.50

16.00

$1 . .30$

10.00

1.50

11.00

2.00

10.00

15.00

.....

.5 .00

.... .

20.00

20.00

16.00

12.00

35.00

.....

12.0()

$+0.00$

17.00

...
$+.111)$

1.50

2.00

1.50

2.00

2.00

1.50

$\stackrel{2.00}{0}$

.....

...

1.00

6.00

1.00

6.00
6.00

10.00

....

1. . 00

15.00

.....

7.00

25.00

30.00

15.00

7.00

10.00

10.00
18.00
1.00

Jack London, salmon with red lines...... . 3.00

1.50 


No. 1
$11 \frac{1}{2}$ in. up
$100^{1000}$

Jewell, dainty pink

Kirchhoff's Violet.

2.00

2.5.00

Le M Foch, light pink.

1.50

$+.00$

Liebesfeuer, liery red.

2.50

$\pm .00$

Maine, white.

1.50

$+.00$

Marie Kundred, wood white.

Mary Pickford, "reant white

$2.5)$

Minuet, lavender, extri

9.00

Miss Spokane, orange .allnon.......... :.00

Mrs. Bothin, ruffled geranium pink. . . . . 2.50

Mrs. Douglas, fine sahmon pink........ t.0(l

Mrs. King, sat

Mrs. Lohrman, ereany shell pink. . . . . . t.0()

Mrs. Morrison, (Kirehhoff's)

Mrs. Norton, lupleblosiom

Mrs. Pendleton, pink with blot.h. . . . . .

Mrs. Peters, laremler pink.

Mrs. Richardson, dark red, slowy.......

Muriel, light blue darker thoat.........

Myra, salmon pink and large...........

Nora, blue.

Orange Glory, ruffled orange.

Paramount, exquisite pink amel ereatut....

Peace, white, lika makings...........

Pink Lily, rulf̈led rose pink. . . . . . . . . .

President Harding, deep tyrian reel. . . . . .

Pride of Lancaster, brilliant orance walmon

Prince of Wales, delicite salmon. . . . . . .

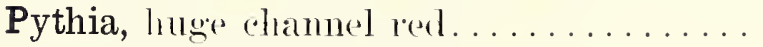

Remembrance, large ruffled sahnon rose. .

Romance, salmon rose borderesl blue. . . . .

Richard Diener, salunon pink. . . . . . . . . .

Rose Ash, aslies of roses. . . . . . . . . . . .

Rosella, larewe old rose. . . . . . . . . . . . .

Rose Glory, ruffled.

Rose 1910, early................... 2.00

Scarlano, laree fine scarlet........... 2.50

Scarlet Wonder, mammoth s(arlet . . . . . 3.50

Shasta, (Kirchhof"s) a real good creamy white. Several 20.00

Sulphur Glow, sulphum rellow . . . . . . . . 3.00

Souvenir, (prinu) vellow............ 2.50

Tycko Zang, rose doree throat white.... 4.00

Uhlan Chief, red. . . . . . . . . . . . . . . . . . 4.00

Virginia, scarlet popular............ 2.00

W. H. Phipps, iridescent pink. . . . . . . . 8.00

Wilbrink, light pink, early. . . . . . . . 2. 2.00

Wm. Badger, light salmon. . . . . . . . . . . 3.00

Yellow Hammer, early vellow. . . . . . . . 2.50

Exhibition Mixed.
1.5. 00

200.00

12.00

....

-). (1)

12.00

....

20.00

......

-) (1) (1)

:36. (10)

$1+.110$

.....

.5 .5110

30.00

$1+.00$

30.00

30.00

......

14.00

$1+.00$

$1+.00$

60.00

$1+.00$

30.00

20.00

$1+.00$

2.0 .00

20.00

30.00

25.00

$30.0(1)$

….

14.00

18.00

30.00

.5 .00

20.00

30.00

30.00

15.00

(i.). .00

14.00

2.5 .00

20.00

18.00
No. 2
$1^{1}+-1^{1}-2$

100

1000

No, 3

100

1000

1.5010 .00

20.00160 .00

1.(1) 9.00

.80

5.00

3.00

2.00

$1+.00$

1.50

10.00

3.00

1.00

8.00

.60

$+.00$

3.00

2.00

1.). 100

1.50

10.10

20.001

․ . $0(0$

2.00

$\therefore .00$

15. 60

27.00

11.00

1.50

$\therefore .00$

2.50

$\therefore .00$

-9). ()0

․). (1)

1.50

1). (1)

$\therefore .010$

2.). 00

:). (1)

-2. . 00

3.00

1.50

……

1.50

10.00

1.50

1). 00

10.00

16.00

1.50

11). 00

2.50

$2 \cdot 2.00$

1.00

7.00

2.00

15.00

2.00

15.00

1.00

6.00

2.50

1.50

10.00

$+.00$

3.00

2.5 .00

2.00

1.). 100

1.50

10.00

2.50

20.00

2.00

16.00

$\therefore .00$

15.00

2.50

3.00

950

2.00

2.50

20.00

2.50

20.00

3.00

20.00

2.00

12.00

2.00

18.00

2.00

16.00

1.50

10.00

1.50

12.00

2.00

$1+.00$

1.00

6.00

2.50

2.). 00

1.50

10. 00

2.00

1.). 00

....

2.00

....

3.00

$1+.00$

10.00

3.00

2.5 .00

1.50

18.00

1.50

2.5 .00

2.00

1.). 00

6.00

10.00

2.00

7.00

1.50

50.00

1. .00

40.00

10.00

5.00

6.00$$
200
$$

1.00

......

1.50

14.00

1.50

10.00

7.00 
